\title{
A Case of Bilateral External Auditory Canal Pyogenic Granuloma in a Pregnant Woman
}

\author{
Kudamo Song ${ }^{1}$, Jun Lee ${ }^{1}$, Mee Ja Park ${ }^{2}$, and Ho Yun Lee ${ }^{1}$ \\ ${ }^{1}$ Departments of Otorhinolaryngology-Head and Neck Surgery, ${ }^{2}$ Pathlogy, Eulji University Medical Center, Eulji University \\ School of Medicine, Daejeon, Korea
}

Received January 1, 2018

Revised February 1, 2018

Accepted February 2, 2018

Address for correspondence Ho Yun Lee, MD, PhD

Department of Otorhinolaryngology-

Head and Neck Surgery,

Eulji University Medical Center,

Eulji University School of Medicine,

95 Dunsanseo-ro, Seo-gu,

Daejeon 35233, Korea

Tel $+82-42-611-3133$

Fax +82-42-611-3135

E-mail hoyun1004@gmail.com
A pyogenic granuloma is one of the most common benign tumors in the head and neck. However, it rarely occurs in the external auditory canal (EAC). We report a case of a pyogenic granuloma that involved the both EAC in a pregnant woman. The clinical manifestation is discussed with a literature review.

J Audiol Otol 2018;22(4):244-247

KEY WORDS: Pyogenic granuloma · External auditory canal · Pregnancy.

\section{Introduction}

A pyogenic granuloma is an acquired benign tumor, principally of the skin and mucosa; it is also termed a capillary hemangioma by reference to the pathological classification. The tumor etiology remains uncertain but may involve frequent ear-picking and changes in hormone levels. The tumors develop principally in the head-and-neck region $(62.4 \%)$, followed by the trunk (19.7\%), upper limbs (12.9\%), and lower limbs (5.0\%) [1]. In the head-and-neck region, most tumors develop in the cheeks and/or oral cavity; rarely, they develop in the external auditory canal (EAC) [1]. In a literature review, we found five case reports of pyogenic granulomas of the EAC dated up to December 2017 (Table 1) [2-6]. Recently, we treated a pregnant woman with a bilateral pyogenic granuloma involving both EACs; herein, we report this case and review the literature.

This is an Open Access article distributed under the terms of the Creative Commons Attribution Non-Commercial License (https://creativecommons.org/licenses/by-nc/4.0/) which permits unrestricted non-commercial use, distribution, and reproduction in any medium, provided the original work is properly cited.

\section{Case Report}

A 30-year-old woman who was 11 weeks pregnant visited our outpatient clinic complaining of persistent bilateral otorrhea and aural fullness irrespective of frequent ear dressing at a local clinic. She had no past medical history except chronic otitis externa diagnosed at the local clinic; she habitually picked her ears. An otoendoscopic examination revealed slightly narrowed EACs with marked S-shaped curvatures and round, reddish pedunculated masses that partially filled both EACs (Fig. 1) and concealed the tympanic membranes. She was advised to undergo an additional radiological evaluation and excisional biopsy as both diagnosis and treatment. However, she refused, insisting that she be treated only after delivery. Thus, we instructed the patient to irrigate her ears with diluted white vinegar and to visit our hospital after delivery. Six months later, she re-visited the outpatient clinic. Temporal bone computed tomography showed that both EACs were blocked with masses having a maximal diameter of $0.5 \mathrm{~cm}$ on the right and $0.4 \mathrm{~cm}$ on the left (Fig. 2A, B). Neither bony erosion of the EACs nor adhesion of the masses to the tympanic membranes could be confirmed. The patient underwent surgical excision with minimal perioperative bleeding. Both masses were 
Table 1. Prior reports of pyogenic granulomas in the external auditory canal

\begin{tabular}{lcclllc}
\hline \multicolumn{1}{c}{ Reference } & Date & Age/sex & Side & Chief complaint & \multicolumn{1}{c}{ Treatment } & Recurrence \\
\hline Lee, et al. [2] & 2008 & $13 / \mathrm{M}$ & Right & Visible mass & Surgical excision & Yes \\
Hsu, et al. [3] & 2008 & $27 / \mathrm{M}$ & Both & Ear fullness & Laser surgery & No \\
Topdag, et al. [4] & 2014 & 19/F & Left & Painful mass & Wall-down mastoidectomy & No \\
Magliulo, et al. [5] & 2014 & $43 / \mathrm{M}$ & Left & Visible mass & Surgical excision & No \\
Dick, et al. [6] & 2016 & 29/F & Right & Ear fullness & Surgical excision & No \\
\hline
\end{tabular}

Fig. 1. Macroscopic otoendoscopic findings. Pinkish, smooth pedunculated masses partially filled both EACs. A: Right EAC, B: Left EAC. EAC: external auditory canal.
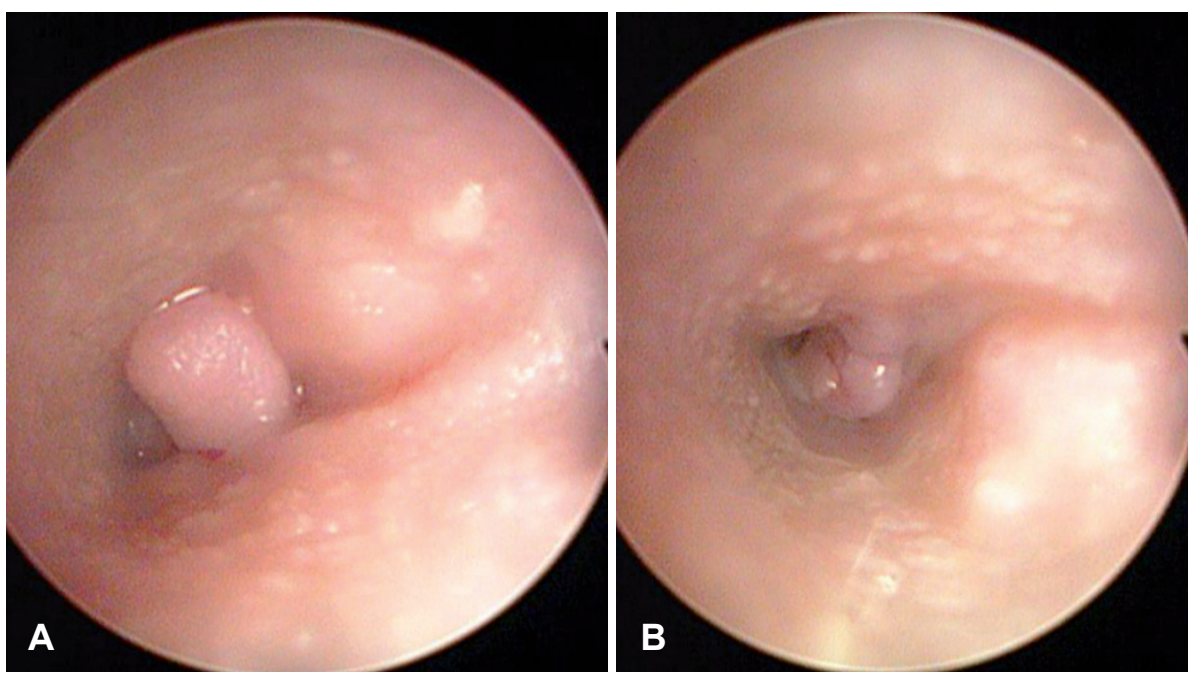

Fig. 2. Temporal bone CT findings and specimen data. The maximal diameters of the masses were, respectively, 0.5 and $0.4 \mathrm{~cm}$. No bony erosion was evident adjacent to the external auditory canal. No clear adhesions between the masses and tympanic membranes were apparent. Black arrows indicate masses blocking both external auditory canal without bony erosion. A, B: Temporal CT, coronal view, C: Right-side specimen, D: Left-side specimen. CT: computed tomography.
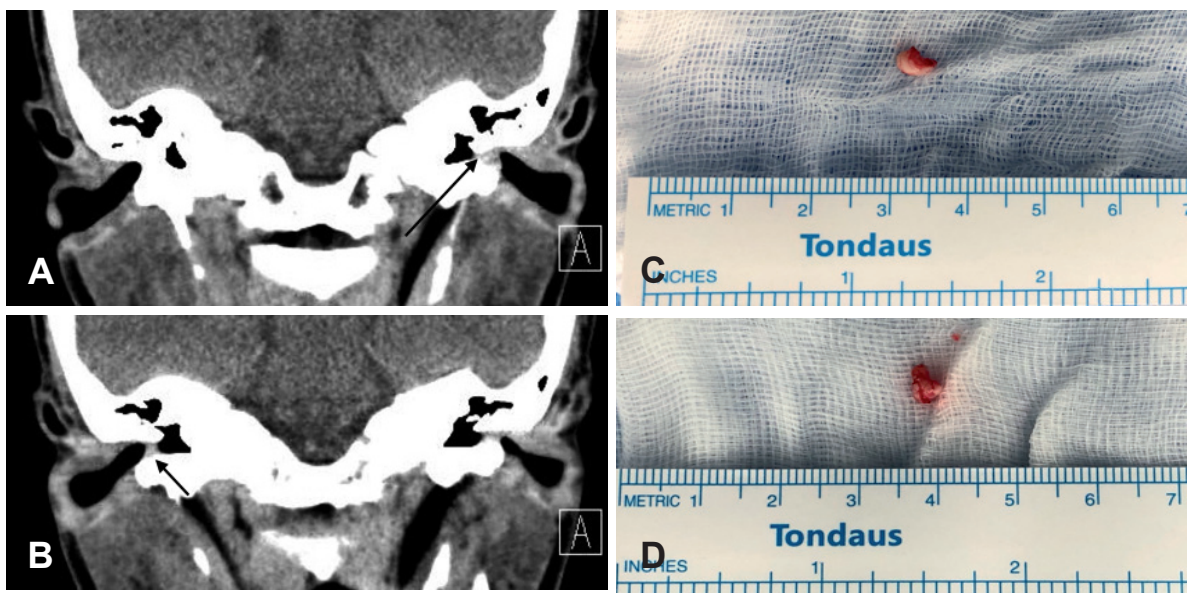

round and firm, and were stalked on the anteroinferior walls of both EACs distant from the tympanic membranes (Fig. $2 \mathrm{C}, \mathrm{D})$. Histopathologically, the tumors were pyogenic granulomas (Fig. 3). No sign of recurrence was noted at the 2-month follow-up exam. This patient has given written informed consent for publication.

\section{Discussion}

Patients with pyogenic granulomas may visit outpatient clinics complaining of pulsatile tinnitus, recurrent otorrhagia, otorrhea, and/or deafness [7]. Common physical findings include a purplish tympanic membrane and pedunculated ear mass. The pathogenesis is unclear, but may feature changes in hormone secretion, increased levels of vascular growth factors, chronic mechanical stimulation or trauma, and/or drug side-effects [8]. During pregnancy, marked increases in the levels of female hormones stimulate the secretion of many growth factors throughout the body [9]. Estrogen acts on macrophages to stimulate the secretion of nerve growth factor, which aids in wound-healing and tissue regeneration when injury is caused by chronic mechanical stimulation. Estrogen 

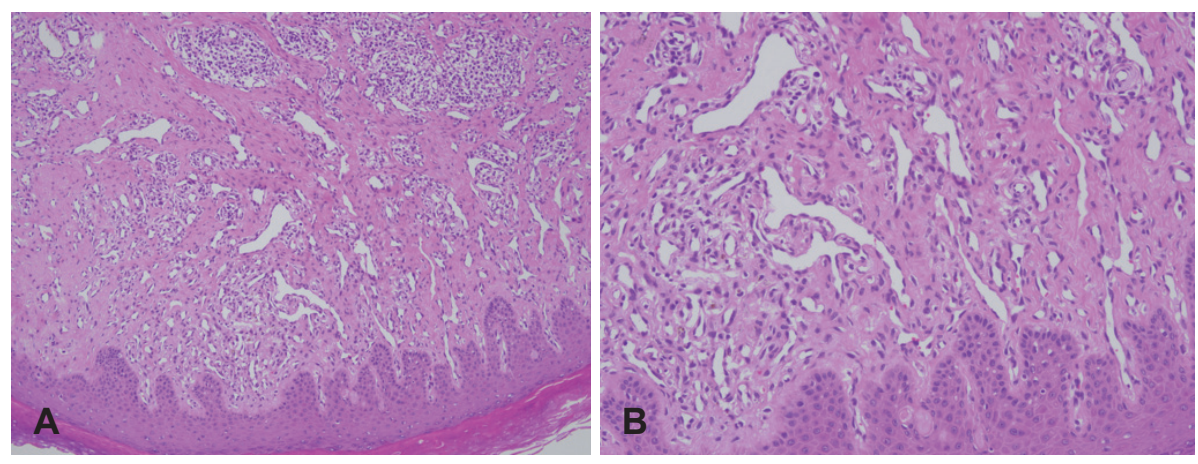

Fig. 3. Histopathological findings. The surfaces of the masses were covered with a keratinized squamous epithelium. The matrices featured capillary beds with abundant vessels and endothelial linings (hematoxylin and eosin staining). A: $\times 100$, B: $\times 200$.

also increases the circulating levels of granulocyte macrophage-colony stimulating factor, basic fibroblast growth factor, and transforming growth factor $\beta-1$, all of which stimulate the growth of granulation tissue [10]. In addition, vascular endothelial growth factor secreted by macrophages stimulates capillary growth and proliferation and may trigger pyogenic granuloma development [10]. Also, drugs such as doxetacel, paclitaxel, and levothyroxine are associated with the occurrence of a pyogenic granuloma, perhaps by exerting toxic effects on tissues and proangiogenic (proliferative) effects on blood vessels [11].

In our case, continuous mechanical stimulation via frequent ear-picking and overgrowth of inflammatory tissue attributable to the increased levels of female hormones during pregnancy might have triggered the development of a pyogenic granuloma in both EACs. The literature contains one case of a pyogenic granuloma in a pregnant woman, but the lesion was unilateral and the patient denied that she engaged in habitual ear-picking [6].

To the best of our knowledge, this is the first report of a bilateral pyogenic granuloma (thus involving both EACs) in a pregnant woman who habitually engaged in chronic ear irritation. A recent study reported that pruritis is common during pregnancy (in up to $38 \%$ of women); ear itching may thus also be common [12]. Clinicians should consider a pyogenic granuloma of the EAC when examining pregnant patients with EAC masses who complain of ear itching.

The treatment of choice is simple surgical excision; other options include curettage with or without cauterization, cryotherapy, and $\mathrm{CO}_{2}$ laser ablation. However, surgical excision is optimal, considering the cost, convenience, and recurrence rate [13]. In case of recurrence, wide excision and cautery may yield success rate [2]. Hsu et al used laser surgery in order to prevent scar formation or unexpected deformity [3]. Canal wall down mastoidectomy was performed to remove a large lesion $\geq 30 \mathrm{~mm}$ in diameter [4].

Characteristic histopathological findings include high-level proliferation of matrix endothelium and capillaries and di- lated blood vessels that are often arranged in lobules. Ulcerative areas are also common; neutrophils may be detected near ulcerated surfaces. These findings are rather specific, making it easy to diagnose a pyogenic granuloma, but a hemangioma also features a lobular arrangement of capillaries; a pyogenic granuloma is thus sometimes misdiagnosed [14].

In summary, we reported the case of a pregnant woman with a bilateral pyogenic granuloma (in both EACs). Chronic irritation caused by ear-picking and hormonal changes may have triggered the condition; surgical excision was essential.

\section{Conflicts of interest}

The authors have no financial conflicts of interest.

\section{REFERENCES}

1) Patrice SJ, Wiss K, Mulliken JB. Pyogenic granuloma (lobular capillary hemangioma): a clinicopathologic study of 178 cases. Pediatr Dermatol 1991;8:267-76.

2) Lee MH, Cho SJ, Lee DH, Cho KJ. A case of pyogenic granuloma of the external auditory canal. J Audiol Otol 2008;12:129-32.

3) Hsu $\mathrm{CH}$, Chen $\mathrm{HC}$, Wang $\mathrm{CH}$. Bilateral external auditory canal pyogenic granuloma. Otolaryngol Head Neck Surg 2008;139:596-7.

4) Topdag M, Mutlu A, Sari F, Ozturk M. An external auditory canal lesion: lobular capillary hemangioma. J Otolaryngol ENT Res 2014; 1:00005.

5) Magliulo G, Iannella G, Gianno F, Re M. Lobular capillary hemangioma and external auditory canal. Otol Neurotol 2014;35:e213-4.

6) Dick DC, Elliott K, Napier S, Adair R. Pregnancy tumour of the external auditory canal: treatment in clinic. BMJ Case Rep [serial online] 2016 Feb 16 [cited 2017 December 25]. Available from: http://doi. org/10.1136/ bcr-2015-213673.

7) Schild C, Kayser G, Aschendorff A, Boedeker CC. Pedunculated capillary hemangioma of the external ear. Otolaryngol Head Neck Surg 2009;140:764-5.

8) Jafarzadeh H, Sanatkhani M, Mohtasham N. Oral pyogenic granuloma: a review. J Oral Sci 2006;48:167-75.

9) Katta R, Bickle K, Hwang L. Pyogenic granuloma arising in portwine stain during pregnancy. Br J Dermatol 2001;144:644-5.

10) Kanda N, Watanabe S. Regulatory roles of sex hormones in cutaneous biology and immunology. J Dermatol Sci 2005;38:1-7.

11) Devillers $C$, Vanhooteghem O, Henrijean $A$, Ramaut M, de la Brassinne M. Subungueal pyogenic granuloma secondary to docetaxel therapy. Clin Exp Dermatol 2009;34:251-2.

12) Szczęch J, Wiatrowski A, Hirnle L, Reich A. Prevalence and relevance of pruritus in pregnancy. Biomed Res Int 2017;2017:4238139. 
13) Lee J, Sinno H, Tahiri Y, Gilardino MS. Treatment options for cutaneous pyogenic granulomas: a review. J Plast Reconstr Aesthet Surg 2011;64:1216-20.

14) Varma S, Gangavati R, Sundaresh KJ, Mallikarjuna R. Lobulated capillary haemangioma: a common lesion in an uncommon site. BMJ Case Rep [serial online] 2013 Feb 15 [cited 2017 December 25]. Available from: http://doi.org/10.1136/bcr-2012-008535. 